\title{
One-body information loss in fermion systems
}

\author{
N. Gigena, R.Rossignoli \\ IFLP-Departamento de Física, Universidad Nacional de La Plata, C.C. 67, La Plata (1900), Argentina
}

\begin{abstract}
We propose an entropic measure of non-classical correlations in general mixed states of fermion systems, based on the loss of information due to the unread measurement of the occupancy of single particle states of a given basis. When minimized over all possible single particle bases, the measure reduces to an entanglement entropy for pure states and vanishes only for states which are diagonal in a Slater determinant basis. The approach is also suitable for states having definite number parity yet not necessarily a fixed particle number, in which case the minimization can be extended to all bases related through a Bogoliubov transformation if quasiparticle mode measurements are also considered. General stationary conditions for determining the optimizing basis are derived. For a mixture of a general pure state with the maximally mixed state, a general analytic evaluation of the present measure and optimizing basis is provided, which shows that non-entangled mixed states may nonetheless exhibit a non-zero information loss.

PACS numbers: 03.67.Mn, 03.65.Ud, 05.30.Fk
\end{abstract}

\section{INTRODUCTION}

Quantification of non-classical correlations in quantum systems is one of the main topics in quantum information theory. Quantum entanglement is the most famous and best studied manifestation of such correlations, mainly because of its central role as a resource for quantum teleportation [1] and quantum computation [2-4]. Nevertheless, quantum entanglement is not the only type of non-classical correlation. It is now well-known that nonentangled mixed states can also exhibit non-classical features, which may be relevant in mixed-state based algorithms such as that of Knill and Laflamme [ [5, [6] . A great effort has therefore been devoted in recent years to understand and quantify the quantumness of correlations 7]. Various measures of these correlations beyond entanglement have then been proposed, which start with the quantum discord [8-11] and the one-way information deficit [12, 13]. The latter has been extended to more general entropic forms [14], as a measure of the information loss due to a local measurement. Various other related measures have also been later introduced $7,15-21]$.

While these correlation measures have been intensively investigated in systems of distinguishable components, less attention has been given to their extension to systems of identical particles [22], and in particular to fermion systems. In these systems particles cannot be accessed individually because of indistinguishability, thus preventing the straightforward extension of the correlation measures defined for systems of distinguishable components, which are based on the tensor product structure of the state space. This property no longer holds for indistinguishable components, turning the characterization of correlations more complex. Various approaches for describing and quantifying entanglement in fermion systems have been introduced, based on quantum correlations and particle-like entanglement [23 31] and also on mode-type entanglement 32 35].

In a previous work [31] we analyzed the problem of quantifying entanglement in pure and mixed states of fermion systems having definite number parity, yet not necessarily a fixed particle number. The approach is based on a consistent definition of the measurement of the occupancy of a single-particle (sp) mode, and of the reduced state of such a mode and its orthogonal complement in the sp Hilbert space $\mathcal{S}$. It leads to an entanglement entropy which is explicitly invariant under particle-hole (and also Bogoliubov) transformations, and which extends previous treatments for states with fixed particle number [23], providing at the same time a link between mode-based and particle based approaches.

In this work we start by considering again the correlations between a sp mode and its orthogonal complement and define an entropic measure that quantifies the loss of information in the state of the system due to the measurement of the occupancy of that mode. Such loss is directly related to the entanglement generated between the system and the measurement device. We then consider the sum over all states on a given basis of $\mathcal{S}$, minimized over all such bases, of this quantity as a measure of the minimum loss of information due to an unread measurement in this basis, extending the minimization to all bases related through a Bogoliubov transformation [36] if the occupancy measurement of quasiparticle modes is also allowed. It is then shown that this measure is a non negative quantity which is zero if and only if the state of the system is a convex combination of Slater determinants in the same sp basis, reducing for pure states to the entanglement entropy defined in [31], in analogy with its counterpart [14] for systems of distinguishable constituents. General stationary conditions for the optimizing basis are also derived. An analytic evaluation of this measure for a mixture of a general pure state with the maximally mixed state is provided, which shows that in this case the optimizing quasiparticles are just those diagonalizing the generalized one-body density matrix, and that the present measure can be non-zero in nonentangled mixed states. Explicit comparison with the fermionic entanglement of formation in a specific case is also made. Two fermion states are as well discussed. 


\section{FORMALISM}

\section{A. Single mode measurement and entanglement entropy}

We first consider a pure state $|\psi\rangle$ of a fermion system with an $n$-dimensional single-particle (sp) Hilbert space $\mathcal{S}$. The system is described by a set of operators $\left\{c_{j}, c_{j}^{\dagger},\right\}$ satisfying the canonical anti-commutation relations

$$
\left\{c_{i}, c_{j}\right\}=0, \quad\left\{c_{i}, c_{j}^{\dagger}\right\}=\delta_{i j},
$$

such that $\left\{|j\rangle=c_{j}^{\dagger}|0\rangle, j=1, \ldots, n\right\}$ is an orthonormal set of one fermion states states $(|0\rangle$ denotes the vacuum of the operators $c_{j}$ ). We will work within a grand canonical context, so the state $|\psi\rangle$ does not necessarily has a definite particle number $N=\sum_{k} c_{k}^{\dagger} c_{k}$. Nonetheless, we will always assume that $|\psi\rangle$ has a definite number parity,

$$
P|\psi\rangle= \pm|\psi\rangle, \quad P=\exp [i \pi N]=\prod_{k}\left(1-2 c_{k}^{\dagger} c_{k}\right),
$$

in agreement with the standard superselection rule 37]. We will also denote by $d$ the dimension of the Fock space of the system.

We now consider a partition $(A, B)$ of a particular basis of $\mathcal{S}$ (see Appendix), where $A$ denotes the single mode or "level" $k$ and $B$ its orthogonal complement. Due to the anticommutation relations, the operators

$$
\Pi_{k}=c_{k}^{\dagger} c_{k}, \quad \Pi_{\bar{k}}=c_{k} c_{k}^{\dagger}, \quad \Pi_{k}+\Pi_{\bar{k}}=1,
$$

constitute a basic set of orthogonal projectors, defining a standard projective measurement on the level $k$. The operator $\Pi_{k}\left(\Pi_{\bar{k}}\right)$ projects the state onto the subspace of states with level $k$ occupied (empty), so the set describes the measurement of the occupancy of this level. The ensuing post measurement states are $\left|\psi_{k}\right\rangle=\Pi_{k}|\psi\rangle / \sqrt{p_{k}}$ and $\left|\psi_{\bar{k}}\right\rangle=\Pi_{\bar{k}}|\psi\rangle / \sqrt{p_{\bar{k}}}$, with $p_{k}=\left\langle c_{k}^{\dagger} c_{k}\right\rangle=\left\langle\psi\left|c_{k}^{\dagger} c_{k}\right| \psi\right\rangle$ and $p_{\bar{k}}=\left\langle c_{k} c_{k}^{\dagger}\right\rangle=1-p_{k}$, such that

$$
|\psi\rangle=\sqrt{p_{k}}\left|\psi_{k}\right\rangle+\sqrt{p_{\bar{k}}}\left|\psi_{\bar{k}}\right\rangle .
$$

For any operator $O_{A}\left(O_{B}\right)$ that depends only on the operators $c_{k}, c_{k}^{\dagger}\left(\left\{c_{j}, c_{j}^{\dagger}, j \neq k\right\}\right)$ we have, using (2),

$$
\begin{aligned}
\left\langle\psi\left|O_{A(B)}\right| \psi\right\rangle & =p_{k}\left\langle\psi_{k}\left|O_{A(B)}\right| \psi_{k}\right\rangle+p_{\bar{k}}\left\langle\psi_{\bar{k}}\left|O_{A(B)}\right| \psi_{\bar{k}}\right\rangle \\
& =\operatorname{Tr} \rho_{A(B)} O_{A(B)},
\end{aligned}
$$

where

$$
\begin{aligned}
& \rho_{A}=p_{k} c_{k}^{\dagger}|0\rangle\left\langle 0\left|c_{k}+p_{\bar{k}}\right| 0\right\rangle\langle 0|, \\
& \rho_{B}=p_{k} c_{k}\left|\psi_{k}\right\rangle\left\langle\psi_{k}\left|c_{k}^{\dagger}+p_{\bar{k}}\right| \psi_{\bar{k}}\right\rangle\left\langle\psi_{\bar{k}}\right| .
\end{aligned}
$$

play the role of reduced states for $A$ and $B$.

As shown in [31], for pure states the mode measurement defined above allows to define an entanglement entropy that quantifies the entanglement between the measured sp mode and its orthogonal complement in $\mathcal{S}$. Indeed, the reduced states (6) -(7) have the same eigenvalues $\left\{p_{k}, p_{\bar{k}}\right\}$, and hence the entanglement entropy for such bipartition is the Shannon entropy of that distribution:

$$
E_{k}(|\psi\rangle)=-p_{k} \log _{2}\left(p_{k}\right)-p_{\bar{k}} \log _{2}\left(p_{\bar{k}}\right) .
$$

The sum of (8) over all states on a given basis of $\mathcal{S}$, minimized over all sp bases, is the one-body entanglement entropy [31]

$$
S^{\mathrm{sp}}(|\psi\rangle)=\operatorname{Min}_{\left\{c_{k}\right\}} \sum_{k}-p_{k} \log _{2} p_{k}-p_{\bar{k}} \log _{2} p_{\bar{k}} .
$$

The minimum in Eq. (9) is reached for those operators $\left\{c_{k}\right\}$ that diagonalize the one-body density matrix $\rho_{i j}^{\mathrm{sp}}=$ $\left\langle c_{j}^{\dagger} c_{i}\right\rangle$, i.e., for those satisfying $\left\langle c_{j}^{\dagger} c_{i}\right\rangle=\lambda_{i} \delta_{i j}$ [31]. Hence,

$$
S^{\mathrm{sp}}(|\psi\rangle)=\operatorname{tr} h\left(\rho^{\mathrm{sp}}\right)
$$

where $h(p)=-p \log _{2} p-(1-p) \log _{2}(1-p)$ and tr the trace in the sp space. It is clear then that $S^{\mathrm{sp}}(|\psi\rangle)=0$ iff there is a sp basis in which the state is written as a Slater determinant, for in that case the eigenvalues of $\rho^{\mathrm{sp}}$ are either 0 or $1\left(\left(\rho^{\mathrm{sp}}\right)^{2}=\rho^{\mathrm{sp}}\right)$. Then Slater determinants are considered here non-correlated states, in agreement with [23].

If quasiparticle modes are to be allowed, then the minimization extends to all quasiparticle bases, i.e., sets of operators $\left\{a_{i}, a_{i}^{\dagger}\right\}$ related to the original fermion operators $\left\{c_{i}, c_{i}^{\dagger}\right\}$ through a Bogoliubov transformation [36]:

$$
a_{i}=\sum_{j} \bar{U}_{j i} c_{j}+V_{j i} c_{j}^{\dagger}
$$

Eq. (11) can be written as

$$
\left(\begin{array}{c}
\boldsymbol{a} \\
\boldsymbol{a}^{\dagger}
\end{array}\right)=\mathcal{W}^{\dagger}\left(\begin{array}{c}
\boldsymbol{c} \\
\boldsymbol{c}^{\dagger}
\end{array}\right), \mathcal{W}=\left(\begin{array}{cc}
U & V \\
\bar{V} & \bar{U}
\end{array}\right),
$$

where the $2 n \times 2 n$ matrix $\mathcal{W}$ should be unitary $\left(U U^{\dagger}+\right.$ $\left.V V^{\dagger}=1, \quad U V^{T}+V U^{T}=0\right)$ in order that $a_{\nu}, a_{\nu}^{\dagger}$ fulfill the fermionic anticommutation relations (1). It can be shown [31] that the minimum is reached for those operators $\left\{a_{\mu}, a_{\mu}^{\dagger}\right\}$ that diagonalize the extended $2 n \times 2 n$ density matrix

$$
\rho^{\mathrm{qsp}}=1-\left\langle\left(\begin{array}{c}
\boldsymbol{c} \\
\boldsymbol{c}^{\dagger}
\end{array}\right)\left(\begin{array}{ll}
\boldsymbol{c}^{\dagger} & \boldsymbol{c}
\end{array}\right)\right\rangle=\left(\begin{array}{cc}
\rho^{\mathrm{sp}} & \kappa \\
-\bar{\kappa} & 1-\bar{\rho}^{\mathrm{sp}}
\end{array}\right)
$$

where $\kappa_{i j}=\left\langle c_{j} c_{i}\right\rangle,-\bar{\kappa}_{i j}=\left\langle c_{j}^{\dagger} c_{i}^{\dagger}\right\rangle$ and $\left(1-\bar{\rho}^{\mathrm{sp}}\right)_{i j}=\left\langle c_{j} c_{i}^{\dagger}\right\rangle$. Eq. (13) is an hermitic matrix which can always be diagonalized by a suitable transformation (12), such that

$$
1-\left\langle\left(\begin{array}{c}
\boldsymbol{a} \\
\boldsymbol{a}^{\dagger}
\end{array}\right)\left(\begin{array}{cc}
\boldsymbol{a} & \boldsymbol{a}^{\dagger}
\end{array}\right)\right\rangle=\mathcal{W}^{\dagger} \rho^{\mathrm{qsp}} \mathcal{W}=\left(\begin{array}{cc}
f & 0 \\
0 & 1-f
\end{array}\right),
$$

with $f_{k l}=f_{k} \delta_{k l}$ and $f_{k}, 1-f_{k}$ the eigenvalues of $\rho^{\mathrm{qsp}}$ (which come in pairs $\left(f_{k}, 1-f_{k}\right)$, with $\left.f_{k} \in[0,1]\right)$, entailing $\left\langle a_{k}^{\dagger} a_{k}\right\rangle=\delta_{k l} f_{k},\left\langle a_{k} a_{l}\right\rangle=0$. The generalized one-body entanglement entropy is therefore

$$
\begin{aligned}
S^{\mathrm{qsp}}(|\psi\rangle) & =-\sum_{k} f_{k} \log _{2} f_{k}+\left(1-f_{k}\right) \log _{2}\left(1-f_{k}\right) \\
& =-\operatorname{tr}^{\prime} \rho^{\mathrm{qsp}} \log _{2} \rho^{\mathrm{qsp}}
\end{aligned}
$$


with $\operatorname{tr}^{\prime}$ the trace in the extended single particle space. Hence, $S^{\mathrm{qsp}}(|\psi\rangle)=0$ iff all eigenvalues $f_{k}$ are 0 or 1, i.e., $\left(\rho^{\mathrm{qsp}}\right)^{2}=\rho^{\mathrm{qSp}}$, which implies that $\rho$ is a vacuum or in general a quasiparticle Slater Determinant.

\section{B. Information loss due to a single mode measurement}

Let us now consider a single mode measurement on a general mixed state $\rho$ of a fermion system (assumed to satisfy $[\rho, P]=0)$. The state after finding that sp state $|k\rangle$ is occupied or empty is $\rho_{k}^{\prime}=\Pi_{k} \rho \Pi_{k} / p_{k}$, or $\rho_{\bar{k}}^{\prime}=$ $\Pi_{\bar{k}} \rho \Pi_{\bar{k}} / p_{\bar{k}}$ respectively, with $p_{k}=\left\langle c_{k}^{\dagger} c_{k}\right\rangle=\operatorname{Tr} \rho c_{k}^{\dagger} c_{k}$ and $p_{\bar{k}}=1-p_{k}$. Therefore, after an unread measurement of that level the state of the system is

$$
\rho^{\prime}(k)=p_{k} \rho_{k}^{\prime}+p_{\bar{k}} \rho_{\bar{k}}^{\prime}=\Pi_{k} \rho \Pi_{k}+\Pi_{\bar{k}} \rho \Pi_{\bar{k}} .
$$

For instance, if $\rho$ is a pure state $|\psi\rangle\langle\psi|$ (Eq. (4)),

$$
\rho^{\prime}(k)=p_{k}\left|\psi_{k}\right\rangle\left\langle\psi_{k}\left|+p_{\bar{k}}\right| \psi_{\bar{k}}\right\rangle\left\langle\psi_{\bar{k}}\right| .
$$

The ensuing reduced state of $A$ is given by (6) while $\rho_{B}=p_{k} c_{k} \rho_{k}^{\prime} c_{k}^{\dagger}+p_{\bar{k}} \rho_{\bar{k}}^{\prime}$. Since $\rho=\rho^{\prime}(k)+\Pi_{k} \rho \Pi_{\bar{k}}+\Pi_{\bar{k}} \rho \Pi_{k}$, the last two terms are lost after such measurement.

The entropy $S\left(\rho^{\prime}(k)\right)=-\operatorname{Tr} \rho^{\prime}(k) \log _{2} \rho^{\prime}(k)$ of the post-measurement state (15) then cannot be lower than the entropy $S(\rho)$ of the original state, due to the information contained in the lost elements. In fact, the eigenvalues of $\rho^{\prime}(k)$ are just the diagonal elements of $\rho$ in a basis different from that of its eigenvectors, and it is well known that any such diagonal is always majorized by the eigenvalues of $\rho[38]$. We have then

$$
S\left(\rho^{\prime}(k)\right) \geq S(\rho),
$$

with equality if and only if $\rho^{\prime}(k)=\rho$. This last condition is obviously equivalent to

$$
\left[\rho, c_{k}^{\dagger} c_{k}\right]=0,
$$

since if $\rho=\rho^{\prime}(k)$, Eq. (18) holds, while if (18) is valid, there is common basis of eigenvectors of $\rho$ and $c_{k}^{\dagger} c_{k}$ and hence $\rho=\rho^{\prime}(k)$. The difference

$$
I^{c_{k}}(\rho)=S\left(\rho^{\prime}(k)\right)-S(\rho)
$$

quantifies then this loss of information. It clearly satisfies $I^{c_{k}}(\rho) \geq 0$, with $I^{c_{k}}(\rho)=0$ iff (18) applies. It is a fermionic version of the information deficit $\Delta^{\rightarrow}(\rho)=$ $S\left(\rho^{\prime}\right)-S(\rho)$ [12], defined for systems of distinguishable constituents (there is here no minimization involved because the occupation of mode $|k\rangle$ is a classical variable). Furthermore, it is worth noting from (15) that

$$
S\left(\rho^{\prime}(k)\right)=S(k)+S(\rho \mid k),
$$

where

$$
S(k)=-p_{k} \log _{2} p_{k}-p_{\bar{k}} \log _{2} p_{\bar{k}}=h\left(p_{k}\right)
$$

is the entropy of mode $k$ and

$$
S(\rho \mid k)=p_{k} S\left(\rho_{k}^{\prime}\right)+p_{\bar{k}} S\left(\rho_{\bar{k}}^{\prime}\right),
$$

is the conditional entropy of the set of remaining modes, given that the state of mode $k$ (occupied or empty) is known. Therefore, the deficit (19) can be written as

$$
S\left(\rho^{\prime}(k)\right)-S(\rho)=S(\rho \mid k)-(S(\rho)-S(k)),
$$

which is a difference of classical-like and quantum conditional entropies with respect to mode $k$ and represents the quantum discord [8] with respect to this mode. Hence, for single-mode measurements the extension of quantum deficit coincides with that of the quantum discord. For a pure state $\rho=|\psi\rangle\langle\psi|, \rho_{k}^{\prime}$ and $\rho_{\bar{k}}^{\prime}$ are both pure (Eq. (16)), implying that $I^{c_{k}}$ becomes coincident with the entanglement entropy (8) of mode $k$ :

$$
I^{c_{k}}(|\psi\rangle)=S(k)=E_{k}(|\psi\rangle) .
$$

The conditional entropy (22) can be interpreted, following the general results of [39], as an entanglement of formation between the set of remaining modes $k^{\prime} \neq k$ and a complementary system (which can also be a set of new fermionic modes) which purifies the whole system (see Appendix, Eq. (A.9)). Besides, the arguments of [13, 16] imply that the information deficit (19) is an indicator of the entanglement generated between the measurement device and the system after a measurement of mode $k$. In fact, by adding a qubit ancilla $C$ in an initial state $|0\rangle$ to the fermionic system and performing the unitary transformation $U=e^{-i \frac{\pi}{2}\left(c_{k} c_{k}^{\dagger}\right) \otimes \sigma_{y}}$, we obtain

$$
U(|\psi\rangle|0\rangle)=\sqrt{p_{k}}\left|\psi_{k}\right\rangle|0\rangle+\sqrt{p_{\bar{k}}}\left|\psi_{\bar{k}}\right\rangle|1\rangle .
$$

Hence, for a general mixed fermion state $\rho$,

$$
\operatorname{Tr}_{C}\left[U(\rho \otimes|0\rangle\langle 0|) U^{\dagger}\right]=\rho^{\prime}(k) .
$$

Therefore $I^{c_{k}}(\rho)$ is the difference between the entropy of the fermionic subsystem $\rho_{F}=\operatorname{Tr}_{C} \rho_{F C}=\rho^{\prime}(k)$ in $\rho_{F C}=U(\rho \otimes|0\rangle\langle 0|) U^{\dagger}$ and that of the whole system, $S\left(\rho_{F C}\right)=S(\rho)$. Such difference (the negative of the conditional entropy $\left.S\left(\rho_{F C}\right)-S\left(\rho_{F}\right)\right)$ can be positive only if $\rho_{F C}$ is entangled, according to the entropic separability criterion [40-42], and is a lower bound to the one-way distillable entanglement [13, 16, 43]. On the other hand, if $\rho^{\prime}(k)=\rho$, such that $p_{k}=0$ or 1 for any eigenstate of $\rho$, then $\rho_{F C}$ is clearly separable and no entanglement is created.

\section{One-body information loss}

We now take the sum, over all the states of a given basis of $\mathcal{S}$, of $I^{c_{k}}$ in (19),

$$
I^{c}(\rho)=\sum_{k} I^{c_{k}}(\rho)=\sum_{k} S\left(\rho^{\prime}(k)\right)-S(\rho),
$$


as a measure of the loss of information due to an unread measurement in this basis. The minimum over all sp bases of this difference,

$$
I^{\mathrm{sp}}(\rho)=\operatorname{Min}_{\boldsymbol{c}} I^{\boldsymbol{c}}(\rho)=\operatorname{Min}_{\left\{c_{k}\right\}} \sum_{k} I^{c_{k}}(\rho),
$$

measures then the minimum loss of information due to such type of measurement. This one-body information loss clearly satisfies $I^{\mathrm{sp}}(\rho) \geq 0$, since it is a sum of nonnegative terms, with $I^{\mathrm{sp}}(\rho)=0$ iff fermion operators $c_{k}$ exist such that $\rho^{\prime}(k)=\rho \forall k$, i.e., iff

$$
\left[\rho, c_{k}^{\dagger} c_{k}\right]=0, k=1, \ldots, n,
$$

which occurs iff $\rho$ is diagonal in a set of Slater determinants $\left\{\prod_{k}\left(c_{k}^{\dagger}\right)^{n_{k}}|0\rangle\right\}$ in the same sp basis (the common eigenvectors of all $c_{k}^{\dagger} c_{k}$ ). Such states include the typical uncorrelated thermal-like states $\rho \propto \exp [-\beta H]$, with $H=\sum_{k} \varepsilon_{k} c_{k}^{\dagger} c_{k}$, but also any convex combination of Slater determinants in the same basis. These combinations play here the role of "classically" correlated states.

Note that if (29) holds, for $k \neq l$ we have $\left\langle c_{k}^{\dagger} c_{l}\right\rangle=$ $\left\langle\left[c_{k}^{\dagger} c_{k}, c_{k}^{\dagger} c_{l}\right]\right\rangle=0$, so that the operators $c_{k}$ diagonalize the sp density matrix: $\left\langle c_{k}^{\dagger} c_{l}\right\rangle=\lambda_{k} \delta_{k l}$. Therefore, even though the operators minimizing $I^{\boldsymbol{c}}(\rho)$ may not diagonalize $\rho^{\mathrm{sp}}$ in general, they will if $I^{\mathrm{sp}}(\rho)=0$. Thus, $I^{\boldsymbol{c}}(\rho)>0$ in all sp bases diagonalizing $\rho^{\mathrm{sp}}$ imply $\mathrm{I}^{\mathrm{sp}}(\rho)>0$.

For a pure state $\rho=|\psi\rangle\langle\psi|$ equation (24) implies

$$
I^{c}(|\psi\rangle)=\sum_{k} S(k)=\sum_{k} h\left(p_{k}\right),
$$

which is just a concave function of the diagonal elements of $\rho^{\mathrm{sp}}$. Therefore, in this case its minimum over all bases is obtained when $p_{k}$ are its eigenvalues, i.e., when the $c_{k}$ 's are the fermion operators diagonalizing $\rho^{\mathrm{sp}}$ :

$$
I^{\mathrm{sp}}(|\psi\rangle)=\operatorname{tr} h\left(\rho^{\mathrm{sp}}\right)=S^{\mathrm{sp}}(|\psi\rangle) .
$$

This result coincides with the entanglement entropy defined in the previous section, in analogy with the information loss for the distinguishable case [14], which also coincides with the entanglement entropy for pure states.

On the other hand, it is seen from (20) that for a general mixed state $\rho, S\left(\rho^{\prime}(k)\right) \geq S(k)=h\left(p_{k}\right)$ and hence,

$$
\underset{\left\{c_{k}\right\}}{\operatorname{Min}} \sum_{k} S\left(\rho^{\prime}(k)\right) \geq \operatorname{tr} h\left(\rho^{\mathrm{sp}}\right) .
$$

As in the case of the one-body entanglement entropy, if quasiparticle mode measurements are to be allowed then the minimization extends to all quasiparticle bases, i.e., sets of operators $\left\{a_{k}, a_{k}^{\dagger}\right\}$ related to the original fermion operators $\left\{c_{i}, c_{i}^{\dagger}\right\}$ through a Bogoliubov transformation (12). It can be seen by repeating the argument used above that this information loss,

$$
I^{\mathrm{qsp}}(\rho)=\operatorname{Min}_{\left\{a_{k}\right\}} \sum_{k}\left[S\left(\rho^{\prime}(k)\right)-S(\rho)\right]
$$

satisfies $I^{\mathrm{qsp}}(\rho) \geq 0$, with equality iff quasiparticle operators $a_{k}$ exist such that

$$
\left[\rho, a_{k}^{\dagger} a_{k}\right]=0, k=1, \ldots, n,
$$

i.e., iff $\rho$ is diagonal in a set of quasiparticle Slater determinants in the same basis (common eigenvectors of all $\left.a_{k}^{\dagger} a_{k}\right)$. Moreover, as this extended minimization includes the previous one as a particular case, we have

$$
I^{\mathrm{qsp}}(\rho) \leq I^{\mathrm{sp}}(\rho) .
$$

For pure states, the minimum $I^{\mathrm{qsp}}$ is obtained for those $a_{k}^{\dagger}$ diagonalizing the extended density matrix (13), which in analogy with (31) yields

$$
I^{\mathrm{qsp}}(|\psi\rangle)=-\operatorname{tr}^{\prime} \rho^{\mathrm{qsp}} \log _{2} \rho^{\mathrm{qsp}}=S^{\mathrm{qsp}}(|\psi\rangle),
$$

where $S^{\text {qsp }}$ is the generalized entanglement entropy (14). As in the previous case, if (34) holds, the operators $a_{k}$ diagonalize $\rho^{\mathrm{qsp}}$, implying that even though such operators may not minimize $I^{\boldsymbol{a}}(\rho)$ in general, they will if $I^{\mathrm{qsp}}(\rho)=0$.

Let us also remark that $I^{\mathrm{sp}}(\rho)$ remains invariant under standard unitary one-body transformations

$$
\rho \rightarrow U \rho U^{\dagger}, \quad U=\exp \left[-\imath t \sum_{i, j} h_{i j} c_{i}^{\dagger} c_{j}\right]
$$

with $h_{i j}=h_{j i}^{*}$, since they just imply a unitary transformation of the fermion operators $\boldsymbol{c}=\left(c_{1}, \ldots, c_{n}\right)^{T}$ :

$$
\boldsymbol{c} \rightarrow U \boldsymbol{c} U^{\dagger}=\exp [\imath t h] \boldsymbol{c}
$$

with $\rho^{\mathrm{sp}} \rightarrow \exp [\imath t h] \rho^{\mathrm{sp}} \exp [-\imath t h]$. These transformations map Slater determinants onto Slater determinants. Similarly, $I^{\mathrm{qsp}}(\rho)$ remains invariant under general one-body transformations

$$
\begin{aligned}
\rho & \rightarrow W \rho W^{\dagger}, W=\exp [-\imath t H] \\
H & =\sum_{i, j} h_{i j} c_{i}^{\dagger} c_{j}+\frac{1}{2} \Delta_{i j}\left(c_{i}^{\dagger} c_{j}^{\dagger}+c_{j} c_{i}\right) \\
& =\frac{1}{2}\left(\boldsymbol{c}^{\dagger} \boldsymbol{c}\right) \mathcal{H}\left(\begin{array}{c}
c \\
\boldsymbol{c}^{\dagger}
\end{array}\right), \quad \mathcal{H}=\left(\begin{array}{cc}
h & \Delta \\
-\Delta^{*} & -h^{*}
\end{array}\right)
\end{aligned}
$$

where $\Delta^{T}=-\Delta$, since they just imply a Bogoliubov transformation of the fermion operators:

$$
\left(\begin{array}{c}
c \\
\boldsymbol{c}^{\dagger}
\end{array}\right) \rightarrow \exp [\imath t \mathcal{H}]\left(\begin{array}{c}
\boldsymbol{c} \\
\boldsymbol{c}^{\dagger}
\end{array}\right)
$$

with $\rho^{\mathrm{qsp}} \rightarrow \exp [\imath t \mathcal{H}] \rho^{\mathrm{qsp}} \exp [-\imath t \mathcal{H}]$. Therefore, evolution under Hamiltonians $H$ of the previous form will not alter the value of $I^{\mathrm{qsp}}(\rho)$.

Finally, it is worth remarking that

$$
I^{\mathrm{qsp}}(\rho)=0,
$$

for any state $\rho$ with support in a two-dimensional sp space $(n=2)$ which commutes with the parity $P$.

Proof: Let

$$
\rho=\sum_{\nu=1,2} q_{\nu}^{-}\left|\psi_{-}^{\nu}\right\rangle\left\langle\psi_{-}^{\nu}\left|+q_{\nu}^{+}\right| \psi_{+}^{\nu}\right\rangle\left\langle\psi_{+}^{\nu}\right|
$$


be the spectral decomposition of such $\rho$, with

$$
\left|\psi_{-}^{\nu}\right\rangle=\left(\alpha_{1}^{\nu} c_{1}^{\dagger}+\alpha_{2}^{\nu} c_{2}^{\dagger}\right)|0\rangle,\left|\psi_{+}^{\nu}\right\rangle=\left(\beta_{1}^{\nu}+\beta_{2}^{\nu} c_{2}^{\dagger} c_{1}^{\dagger}\right)|0\rangle,
$$

its odd and even parity orthogonal eigenstates $\left(\alpha_{j}^{2}=\right.$ $\left.(-)^{j} \bar{\alpha}_{3-j}^{1}, \beta_{j}^{2}=(-)^{j} \bar{\beta}_{3-j}^{1}\right)$. It is easily seen that these states can be all written as 0,1 or 2-quasiparticle states in a common basis. In fact, $\left\langle c_{2}^{\dagger} c_{1}\right\rangle=\left(q_{1}^{-}-q_{2}^{-}\right) \bar{\alpha}_{2}^{1} \alpha_{1}^{1}$, $\left\langle c_{2}^{\dagger} c_{1}^{\dagger}\right\rangle=\left(q_{1}^{+}-q_{2}^{+}\right) \bar{\beta}_{2}^{1} \beta_{1}^{1}$. Hence, in terms of the quasiparticle operators $a_{k}$ that diagonalize $\rho^{\mathrm{qsp}}\left(\left\langle a_{2}^{\dagger} a_{1}\right\rangle=\right.$

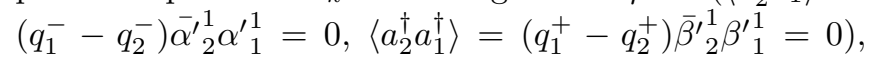
necessarily $\left|\psi_{-}^{1}\right\rangle \propto a_{1}^{\dagger}\left|0^{\prime}\right\rangle,\left|\psi_{-}^{2}\right\rangle \propto a_{2}^{\dagger}\left|0^{\prime}\right\rangle,\left|\psi_{+}^{1}\right\rangle \propto\left|0^{\prime}\right\rangle$ and $\left|\psi_{+}^{2}\right\rangle \propto a_{2}^{\dagger} a_{1}^{\dagger}\left|0^{\prime}\right\rangle$ if $q_{1}^{ \pm} \neq q_{2}^{ \pm}$, with $\left|0^{\prime}\right\rangle$ the vacuum of these quasiparticles (of the same parity as $|0\rangle$ ). And in case of degeneracy we may always choose the eigenstates $\left|\psi_{ \pm}^{\nu}\right\rangle$ of the previous forms. Hence $\rho$ is always diagonal in a quasiparticle Slater determinant basis, implying $I^{\mathrm{qsp}}(\rho)=0$.

Note, however, that $I^{\mathrm{sp}}(\rho)>0$ unless $[\rho, N]=0$, i.e., $\left\langle c_{2}^{\dagger} c_{1}^{\dagger}\right\rangle=0$, and that $\rho$ is not necessarily of the uncorrelated form $\propto \exp \left[-\sum_{\nu=1,2} \varepsilon_{\nu} a_{\nu}^{\dagger} a_{\nu}\right]$ unless $\left\langle a_{1}^{\dagger} a_{1} a_{2}^{\dagger} a_{2}\right\rangle=$ $\left\langle a_{1}^{\dagger} a_{1}\right\rangle\left\langle a_{2}^{\dagger} a_{2}\right\rangle$, i.e., $q_{2}^{+}=\left(q_{1}^{-}+q_{2}^{+}\right)\left(q_{2}^{-}+q_{2}^{+}\right)$.

On the other hand, if the support is a three dimensional sp space $(n=3)$, then $I^{\mathrm{qsp}}(\rho)=0$ for any $\rho$ of definite parity (but not for any $\rho$ commuting with $P$ ). Proof: Let $\rho=\sum_{\nu=1}^{4} q_{\nu}\left|\psi^{\nu}\right\rangle\left\langle\psi^{\nu}\right|$ be the spectral decomposition of $\rho$. If its eigenstates have all odd parity, i.e.

$$
\left|\psi^{\nu}\right\rangle=\left(\sum_{j=1}^{3} \alpha_{j}^{\nu} c_{j}^{\dagger}+\alpha_{4}^{\nu} c_{3}^{\dagger} c_{2}^{\dagger} c_{1}^{\dagger}\right)|0\rangle, \quad \nu=1, \ldots, 4,
$$

then $\left\langle c_{j}^{\dagger} c_{k}\right\rangle=\sum_{\nu} q_{\nu}\left(\bar{\alpha}_{j}^{\nu} \alpha_{k}^{\nu}+\delta_{j k}\left|\alpha_{4}^{\nu}\right|^{2}\right),\left\langle c_{j}^{\dagger} c_{k}^{\dagger}\right\rangle=$ $-\sum_{\nu, l} q_{\nu} \epsilon_{j k l} \bar{\alpha}_{4}^{\nu} \alpha_{l}^{\nu}$, with $\epsilon_{j k l}$ the fully antisymmetric tensor. Hence, if expressed in terms of the quasiparticle operators $a_{k}$ that diagonalize $\rho^{\mathrm{qsp}}\left(c_{j}^{\dagger} \rightarrow a_{j}^{\dagger},|0\rangle \rightarrow\left|0^{\prime}\right\rangle\right.$, $\alpha_{j}^{\nu} \rightarrow \alpha^{\prime \nu}{ }_{j}$, with $\left.\left\langle a_{j}^{\dagger} a_{k}\right\rangle=f_{j} \delta_{j k},\left\langle a_{j}^{\dagger} a_{k}^{\dagger}\right\rangle=0\right)$, the previous relations together with $\left\langle\psi^{\nu} \mid \psi^{\nu^{\prime}}\right\rangle=\sum_{\mu=1}^{4}{\overline{\alpha^{\prime}}}_{\mu}^{\nu} \alpha^{\prime \nu^{\prime}}=\delta^{\nu \nu^{\prime}}$ imply, for distinct $q^{\nu}$ s, $\alpha^{\prime \nu}{ }_{\mu} \propto \delta_{\mu}^{\nu}$, i.e. $\left|\psi^{\nu}\right\rangle \propto a_{\nu}^{\dagger}\left|0^{\prime}\right\rangle$, $\nu=1,2,3$ and $\left|\psi^{4}\right\rangle \propto a_{3}^{\dagger} a_{2}^{\dagger} a_{1}^{\dagger}\left|0^{\prime}\right\rangle$. Hence $\rho$ is diagonal in a quasiparticle Slater determinant basis and $I^{\mathrm{qsp}}(\rho)=0$ (but $I^{\mathrm{sp}}(\rho) \neq 0$ if $[\rho, N] \neq 0$ ). The same procedure can be applied for an even parity $\rho$ (which can be recast in the previous odd-parity form after a particle-hole transformation $c_{i}^{\dagger} \rightarrow c_{i}$ of one of the operators).

Note, however, that if $\rho$ contains eigenstates of different parity, $I^{\mathrm{qsp}}(\rho)$ can be positive since the fermion quasiparticle operators of the normal form for each parity will not coincide in general.

\section{General stationary condition}

Let us now derive the general stationary equations that must be satisfied by the set of operators $\left\{a_{k}\right\}$ minimizing the generalized one-body information loss (33). After a measurement of the occupancy of a corresponding level $k$, the ensuing state $\rho^{\prime}(k)$, Eq. (15), has eigenstates $\left|\phi_{k(\bar{k})}^{\mu}\right\rangle$ with eigenvalues

$$
q_{k(\bar{k})}^{\mu}=\left\langle\phi_{k(\bar{k})}^{\mu}|\rho(k)| \phi_{k(\bar{k})}^{\mu}\right\rangle=\left\langle\phi_{k(\bar{k})}^{\mu}|\rho| \phi_{k(\bar{k})}^{\mu}\right\rangle .
$$

Consider now a small variation of the measurement basis determined by a general one-body transformation

$$
W=\exp [-\imath \epsilon H] \approx 1-\imath \epsilon H,
$$

with $H$ of the general form (39)-(40). We then have $\delta q_{k(\bar{k})}^{\mu}=-\imath \epsilon\left\langle\phi_{k(\bar{k})}^{\mu}|[\rho, H]| \phi_{k(\bar{k})}^{\mu}\right\rangle$ up to lowest order in $\epsilon$, which implies, for the information loss (33),

$$
\begin{aligned}
\delta I^{\mathrm{qsp}}(\rho) & =\sum_{k, \mu} f^{\prime}\left(q_{k}^{\mu}\right) \delta q_{k}^{\mu}+f^{\prime}\left(q_{\bar{k}}^{\mu}\right) \delta q_{\bar{k}}^{\mu} \\
& =-\imath \epsilon \operatorname{Tr}\left(\left[\sum_{k} f^{\prime}\left(\rho^{\prime}(k)\right), \rho\right] H\right),
\end{aligned}
$$

where $f^{\prime}(\rho)=-\log _{2} \rho$. The condition $\delta I^{\mathrm{qsp}}(\rho)=0$ for arbitrary $H$ then leads to the stationary equations $\operatorname{Tr}\left[\sum_{k} f^{\prime}\left(\rho^{\prime}(k)\right), \rho\right] c_{i}^{\dagger} c_{j}=0, \operatorname{Tr}\left[\sum_{k} f^{\prime}\left(\rho^{\prime}(k)\right), \rho\right] c_{i}^{\dagger} c_{j}^{\dagger}=0$ $\forall i, j$, which reduce to

$$
\begin{aligned}
& \operatorname{Tr} \rho\left[f^{\prime}\left(\rho^{\prime}(k)\right)+f^{\prime}\left(\rho^{\prime}(l)\right), a_{k}^{\dagger} a_{l}\right]=0, \\
& \operatorname{Tr} \rho\left[f^{\prime}\left(\rho^{\prime}(k)\right)+f^{\prime}\left(\rho^{\prime}(l)\right), a_{k}^{\dagger} a_{l}^{\dagger}\right]=0,
\end{aligned}
$$

$\forall k \neq l$ when expressed in terms of the quasiparticle operators determining the measurement basis $\left(\left[\rho^{\prime}(k), a_{k}^{\dagger} a_{k}\right]=\right.$ $0 \forall k)$, since $\operatorname{Tr}\left[f^{\prime}(\rho(k)), \rho\right] a_{j}^{\dagger} a_{l}=0$ if $j \neq k \neq l$, and also if $j=l=k$. In the case of $I^{\mathrm{sp}}(\rho)$, just eq. (44) is to be considered $(\Delta=0$ in $H)$.

For a pure state $\rho=|\psi\rangle\langle\psi|$, Eqs. (44)-(45) become

$$
\begin{gathered}
{\left[g\left(p_{k}\right)-g\left(p_{\bar{k}}\right)-g\left(p_{l}\right)+g\left(p_{\bar{l}}\right)\right]\left\langle\psi\left|a_{k}^{\dagger} a_{l}\right| \psi\right\rangle=0,} \\
{\left[g\left(p_{k}\right)-g\left(p_{\bar{k}}\right)+g\left(p_{l}\right)-g\left(p_{\bar{l}}\right)\right]\left\langle\psi\left|a_{k}^{\dagger} a_{l}^{\dagger}\right| \psi\right\rangle=0,}
\end{gathered}
$$

for $k \neq l$, where $g(p)=f^{\prime}(p)$ and $p_{k(\bar{k})}$ are the probabilities of finding state $k$ occupied (empty) in $|\psi\rangle$. It is then verified that they are fulfilled by those $a_{k}$ diagonalizing the extended density matrix (13) $\left(\left\langle a_{k}^{\dagger} a_{l}\right\rangle=\left\langle a_{k}^{\dagger} a_{l}^{\dagger}\right\rangle=0\right)$.

\section{E. Generalized one-body information loss}

We may directly extend all previous considerations to more general entropic forms. We first consider the generalized trace form entropies [42, 44]

$$
S_{f}(\rho)=\operatorname{Tr} f(\rho)
$$

where $f:[0,1] \rightarrow \mathbb{R}$ is a smooth strictly concave real function satisfying $f(0)=f(1)=0$. For $f(\rho)=$ $-\rho \log _{2} \rho, S_{f}(\rho)$ becomes the von Neumann entropy $S(\rho)$, whereas for $f(\rho)=2 \rho(1-\rho)$, it becomes the quadratic entropy $S_{2}(\rho)$, which is just a decreasing function of the purity $\operatorname{Tr} \rho^{2}$ and corresponds to the linear approximation 
$-\rho \ln \rho \approx \rho(1-\rho)$. It does not require the explicit knowledge of the eigenvalues of $\rho$, being then easier to determine than $S(\rho)$ [45, 46]. Moreover, the associated generalization of the one-body entanglement entropy (14), $S_{f}^{\mathrm{qsp}}(|\psi\rangle)=\operatorname{tr}^{\prime} f\left(\rho^{\prime \mathrm{qsp}}\right)$ [31] , becomes

$$
S_{2}^{\mathrm{qsp}}(|\psi\rangle)=2 \operatorname{tr}^{\prime} \rho^{\mathrm{qsp}}\left(1-\rho^{\mathrm{qsp}}\right)=4 \sum_{k} f_{k}\left(1-f_{k}\right),
$$

which is just the sum of the fluctuations of the occupancies of all single quasiparticle levels. More generally, these entropies include the family of Tsallis entropies [47], obtained for $f(\rho)=\left(\rho-\rho^{q}\right) /\left(1-2^{1-q}\right)$ with $q>0$, $q \neq 1$, which become proportional to the von Neumann and quadratic entropies for $q \rightarrow 1$ and $q=2$ respectively. We use here the normalization $\operatorname{Tr} f(\rho)=1$ for a maximally mixed single qubit state $\rho$.

A function $f$ defined with the properties stated above ensures that all entropies $S_{f}(\rho)$ satisfy [42, 44] i) $S_{f}(\rho) \geq$ $0 \forall \rho$, with $S_{f}(\rho)=0$ iff $\rho^{2}=\rho$, ii) $S_{f}\left(\sum_{i} q_{i} \rho_{i}\right) \geq$ $\sum_{i} q_{i} S_{f}\left(\rho_{i}\right)$ for $q_{i} \geq 0, \sum_{i} q_{i}=1$ (concavity) and iii),

$$
\rho^{\prime} \prec \rho \Rightarrow S_{f}\left(\rho^{\prime}\right) \geq S_{f}(\rho),
$$

where $\rho^{\prime} \prec \rho$ indicates that the sorted set $\left\{q_{i}^{\prime}\right\}$ of eigenvalues of $\rho^{\prime}\left(q_{i}^{\prime}>q_{j}^{\prime}\right.$ if $\left.i<j\right)$ is majorized [14, 38, 42] by the sorted set $\left\{q_{i}\right\}$ of eigenvalues of $\rho$ :

$$
\rho^{\prime} \prec \rho \Leftrightarrow \sum_{i=1}^{k} q_{i}^{\prime} \leq \sum_{i=1}^{k} q_{i}, \quad k=1, \ldots, d
$$

with $\sum_{i=1}^{d} q_{i}^{\prime}=\sum_{i=1}^{d} q_{i}$. Therefore, $S_{f}(\rho)$ increases with increasing mixedness of $\rho$.

This last property allows the straightforward extension of the results of the previous section to the present more general entropic forms. Indeed, $\rho^{\prime}(k)$ in (15) is majorized by the original state $\rho, \rho^{\prime}(k) \prec \rho$, implying $S_{f}\left(\rho^{\prime}(k)\right) \geq$ $S_{f}(\rho)$ and hence

$$
I_{f}^{c_{k}}(\rho) \equiv S_{f}\left(\rho^{\prime}(k)\right)-S_{f}(\rho) \geq 0,
$$

with $I_{f}^{c_{k}}(\rho)=0$ iff $\rho^{\prime}(k)=\rho$. Eq. (51) is a measure of the information loss due to the unread measurement of the occupation of sp state $k$, and is also an indicator of the entanglement generated between the system and measurement device, according to the generalized entropic separability criterion [42]. By summing over all states in a basis and minimizing over all possible bases of $\mathcal{S}$ we obtain

$$
I_{f}^{\mathrm{sp}}(\rho)=\operatorname{Min}_{\left\{c_{k}\right\}} \sum_{k} I_{f}^{c_{k}}=\operatorname{Min}_{\left\{c_{k}\right\}} \sum_{k} S_{f}\left(\rho^{\prime}(k)\right)-S_{f}(\rho),
$$

which satisfies $I_{f}^{\mathrm{sp}}(\rho) \geq 0$, with $I_{f}^{\mathrm{sp}}(\rho)=0$ iff $\rho$ is diagonal in a set of Slater Determinants in the same sp basis $\left(\left[\rho, c_{k}^{\dagger} c_{k}\right]=0 \forall k\right)$. The minimization may again be extended to all quasiparticle bases through a Bogoliubov transformation, leading to the quantity

$$
I_{f}^{\mathrm{qsp}}(\rho)=\operatorname{Min}_{\left\{a_{k}\right\}} \sum_{k} S_{f}\left(\rho^{\prime}(k)\right)-S_{f}(\rho),
$$

which satisfies $0 \leq I_{f}^{\mathrm{qsp}}(\rho) \leq I_{f}^{\mathrm{sp}}(\rho)$, with $I_{f}^{\mathrm{qsp}}(\rho)=0$ iff $\rho$ is diagonal in a basis of quasiparticle Slater determinants $\left(\left[\rho, a_{k}^{\dagger} a_{k}\right]=0 \forall k\right)$. For pure states $\rho^{2}=\rho$, $I_{f}^{\mathrm{qsp}}(\rho)=S_{f}\left(\rho^{\mathrm{qsp}}\right)$ becomes the generalized entropy of the extended one-body density matrix. Let us remark that the general stationary conditions (44)-(47) remain valid for the present generalization, with $f^{\prime}$ denoting now the derivative of the entropic function $f$.

Eq. (50) remains also valid for Schur-concave functions of $\rho$ 38, which include, in particular, increasing functions of the previous entropies $S_{f}(\rho)$. An example is provided by the quantum version of the Renyi entropies [48],

$$
S_{q}^{R}(\rho)=\frac{\log _{2}\left(\operatorname{Tr} \rho^{q}\right)}{1-q}=\frac{\log _{2}\left[1-\left(1-2^{1-q}\right) S_{q}(\rho)\right]}{1-q},
$$

where $q>0, q \neq 1$, which are just increasing functions of the Tsallis entropies $S_{q}(\rho)$ and approach the von Neumann entropy for $q \rightarrow 1$. The definition of information loss straightforwardly extends to these entropies. The logarithm in (54) implies additivity, i.e., $S_{q}^{R}(\rho \otimes \sigma)=S_{q}^{R}(\rho)+S_{q}^{R}(\sigma)$, which ensures that the addition of an uncorrelated ancilla to the system $(\rho \rightarrow \rho \otimes \sigma)$ has no effect on the associated information deficit [49] $I_{R_{q}}(\rho)=\sum_{k} S_{q}^{R}\left(\rho^{\prime}(k)\right)-S_{q}^{R}(\rho)$. Nonetheless, the optimization problem for $I_{R_{q}}(\rho)$ is the same as that for $I_{q}(\rho)=\sum_{k} S_{q}\left(\rho^{\prime}(k)\right)-S_{q}(\rho)$.

We finally mention that for fermions we may consider yet another way of adding an ancilla to our system $S$, by expanding its sp Hilbert space $\mathcal{S} \rightarrow \mathcal{S} \oplus \mathcal{A}$. A non correlated state of the system $S+A$ will then have the form $\rho_{S} \rho_{A}$, where $\rho_{A}$ and $\rho_{B}$ involve creation and annihilation operators of single particle states in $\mathcal{S}$ and $\mathcal{A}$ respectively (see Appendix). We have $\operatorname{Tr} \rho_{S} \rho_{A}=\operatorname{Tr} \rho_{S} \operatorname{Tr} \rho_{A}$ if traces are taken in a grand canonical ensemble (as we are here assuming), and hence $S_{q}^{R}\left(\rho_{S} \rho_{A}\right)=S_{q}^{R}\left(\rho_{S}\right)+S_{q}^{R}\left(\rho_{A}\right)$.

\section{APPLICATION}

\section{A. Mixture of pure state plus maximally mixed state}

Let us now consider the mixture

$$
\rho=w|\psi\rangle\langle\psi|+\frac{1-w}{d} I_{d},
$$

with $0 \leq w \leq 1$ and $d$ the dimension of the state space. After an unread measurement of mode $|k\rangle$ the state reads

$$
\rho^{\prime}(k)=w\left(p_{k}\left|\psi_{k}\right\rangle\left\langle\psi_{k}\left|+p_{\bar{k}}\right| \psi_{\bar{k}}\right\rangle\left\langle\psi_{\bar{k}}\right|\right)+\frac{1-w}{d} I_{d}
$$

with $p_{k(\bar{k})}=\left\langle c_{k}^{\dagger} c_{k}\right\rangle\left(\left\langle c_{k} c_{k}^{\dagger}\right\rangle\right)$ the probability of finding mode $k$ occupied (empty) in $|\psi\rangle$. Its eigenvalues are 


$$
\begin{aligned}
& q_{k(\bar{k})}=w p_{k(\bar{k})}+\frac{1-w}{d} \text { and } \frac{1-w}{d}, \text { so that } \\
& \begin{aligned}
S_{f}\left(\rho^{\prime}(k)\right) & =f\left(q_{k}\right)+f\left(q_{\bar{k}}\right)+(d-2) f\left(\frac{1-w}{d}\right), \\
I_{f}^{c}(\rho) & =\sum_{k}\left[S\left(\rho^{\prime}(k)\right)-S(\rho)\right] \\
& =\sum_{k}\left[f\left(q_{k}\right)+f\left(q_{\bar{k}}\right)-f\left(w+\frac{1-w}{d}\right)-f\left(\frac{1-\psi}{d}(5) \phi\right)\right.
\end{aligned}
\end{aligned}
$$

We now show that the minimum of $I_{f}^{c}(\rho)$ over all sp basis of $\mathcal{S}$ is reached for the operators $\left\{c_{k}^{\prime}\right\}$ that diagonalize the one-body density matrix $\rho_{i j}^{\mathrm{sp}}=\left\langle c_{j}^{\dagger} c_{i}\right\rangle$, while the minimum over all quasiparticle basis is attained for those $\left\{a_{k}\right\}$ diagonalizing the corresponding extended one-body density matrix $\rho^{\mathrm{qsp}}$.

Proof. Denoting with $\left\{\lambda_{k}=\left\langle c^{\prime \dagger} c_{k}^{\prime}\right\rangle\right\}$ the set of eigenvalues of the one-body density matrix $\rho^{\text {sp }}$, such that $\left\langle c^{\prime \dagger} c^{\prime}{ }_{j}\right\rangle=\lambda_{k} \delta_{k j}$, this distribution majorizes any other diagonal of the matrix, implying $\left\{p_{k}=\left\langle c_{k}^{\dagger} c_{k}\right\rangle\right\} \prec\left\{\lambda_{k}\right\}$ for the sorted sets. Hence, $\left\{q_{k}\right\} \prec\left\{q_{k}^{\prime}\right\}$ if $q_{k}^{\prime}=w \lambda_{k}+\frac{1-w}{d}$ and $0 \leq w \leq 1$, and also $\left\{q_{\bar{k}}\right\} \prec\left\{q_{\bar{k}}^{\prime}\right\}$ if $q_{\bar{k}}^{\prime}=w \lambda_{\bar{k}}+\frac{1-w}{d}$, since $\left\{p_{k}\right\} \prec\left\{\lambda_{k}\right\}$ implies $\left\{p_{\bar{k}}=1-p_{k}\right\} \prec\left\{\lambda_{\bar{k}}=1-\lambda_{k}\right\}$. Therefore, Eq. (50) leads to

$$
\sum_{k} f\left(q_{k}\right)+f\left(q_{\bar{k}}\right) \geq \sum_{k} f\left(q_{k}^{\prime}\right)+f\left(q_{\bar{k}}^{\prime}\right),
$$

implying $I_{f}^{\mathrm{sp}}(\rho)=\operatorname{Min}_{\boldsymbol{c}} I_{f}^{c}(\rho)=I_{f}^{\boldsymbol{c}^{\prime}}(\rho)$. This result also follows directly from the concavity of $f$ and the relation $q_{k(\bar{k})}=\sum_{k^{\prime}}\left|U_{k k^{\prime}}\right|^{2} q_{k^{\prime}\left(\bar{k}^{\prime}\right)}^{\prime}$, with $U$ the unitary matrix diagonalizing $\rho^{\mathrm{sp}}$.

When quasiparticles are also considered, we note that both $p_{k}$ and $p_{\bar{k}}=1-p_{k}$ are diagonal elements of $\rho^{\mathrm{qsp}}$, so that the enlarged sorted set $\left\{p_{k}, p_{\bar{k}}\right\}$ is majorized by the whole sorted set $\left\{f_{k}=\left\langle a_{k}^{\dagger} a_{k}\right\rangle, f_{\bar{k}}=1-f_{k}\right\}$ of eigenvalues of $\rho^{\mathrm{qsp}}\left(\left\langle a_{k}^{\dagger} a_{l}\right\rangle=\delta_{k l} f_{k},\left\langle a_{k}^{\dagger} a_{l}^{\dagger}\right\rangle=0\right)$. Therefore, $\left\{q_{k}, q_{\bar{k}}\right\} \prec\left\{q^{\prime}{ }_{k}, q^{\prime}{ }_{k}\right\}$ for $q^{\prime}{ }_{k(\bar{k})}=w f_{k(\bar{k})}+\frac{1-w}{d}$, implying Eq. (59) and hence $I_{f}^{\text {qsp }}(\rho)=I_{f}^{a}(\rho)$. This result also follows from the relation $q_{k}=\sum_{k^{\prime}}\left|W_{k k^{\prime}}\right|^{2} q_{k^{\prime}}^{\prime}$ between the elements of the enlarged sets, with $W$ the matrix diagonalizing $\rho^{\mathrm{qsp}}$.

These results are valid for the von Neumann entropy as well as for the generalized entropic forms $S_{f}$, and are evidently in agreement with the stationary conditions (44)(45), since for the state (55) they become proportional to Eqs. (46)-(47). They also hold if $\rho$ has a definite parity $P$, i.e., if $I_{d}$ stands for the projector onto the same parity as that of $|\psi\rangle$, in which case $d \rightarrow d / 2$ in (55)-(59). And if $|\psi\rangle$ has definite fermion number $N$, they are also valid in a canonical ensemble, with $d \rightarrow\left(\begin{array}{c}n \\ N\end{array}\right)$.

The previous arguments also imply that if $\left|\psi^{\prime}\right\rangle$ is a state whose one body density matrix is majorized by that of $|\psi\rangle, \rho^{\prime \text { qsp }} \prec \rho^{\mathrm{qsp}}$, and $\rho^{\prime}=w\left|\psi^{\prime}\right\rangle\left\langle\psi^{\prime}\right|+(1-w) I_{d} / d$, then

$$
I_{f}^{\mathrm{qsp}}\left(\rho^{\prime}\right) \geq I_{f}^{\mathrm{qsp}}(\rho)
$$

$\forall w \in[0,1]$ and $\forall S_{f}$. This general inequality reflects the rigorously stronger entanglement of $\left|\psi^{\prime}\right\rangle$, in the sense that $S_{f}^{\mathrm{qsp}}\left(\left|\psi^{\prime}\right\rangle\right)=S_{f}\left(\rho^{\prime \mathrm{qsp}}\right) \geq S_{f}\left(\rho^{\mathrm{qsp}}\right)$ for all entropies $S_{f}$ if $\rho^{\prime \text { qsp }} \prec \rho^{\text {qsp }}$, and indicates that the value of $I_{f}^{\text {qsp }}$ in the mixture (55) is indeed driven by the entanglement of the pure state. The same relation holds for $I_{f}^{\mathrm{sp}}$ if $\rho^{\text {sp }} \prec \rho^{\mathrm{sp}}$.

Let us point out that $I_{f}^{\mathrm{qsp}}(\rho)$ is a strictly increasing function of $w$ for any concave $f$, with $I_{f}^{\text {qsp }}(\rho)>0$ for any $w>0$. From Eq. (58) it is seen that it exhibits for small $\omega$ a universal initial quadratic increase, given by

$$
I_{f}^{\mathrm{qsp}}(\rho) \approx w^{2}\left|f^{\prime \prime}\left(d^{-1}\right)\right| \sum_{k} f_{k}\left(1-f_{k}\right),
$$

with $f_{k}$ the eigenvalues of the $\rho^{\text {qsp }}$ determined by $|\psi\rangle$, which is just proportional to the quadratic entanglement of $|\psi\rangle$, Eq. (49). For $I_{f}^{\mathrm{sp}}(\rho)$ we should just replace $f_{k}$ by the eigenvalues $\lambda_{k}$ of $\rho^{\mathrm{sp}}$. In the case of the quadratic entropy $S_{2}$, Eq. (61) is of course exact $\forall w \in[0,1]$ and independent of $d\left(\left|f^{\prime \prime}\left(d^{-1}\right)\right|=4\right)$.

Previous results are then similar to those obtained for distinguishable bipartite quantum systems [14], where the role played here by the basis diagonalizing $\rho^{\text {qsp }}$ corresponds there to the local part of the Schmidt basis of the pertinent pure state.

\section{B. The case of four single particle levels}

We now focus on the special case of a fermion system with $n=\operatorname{dim}(\mathcal{S})=4$, where the entanglement of formation for general states can be analytically evaluated 23, 31]. For simplicity we will consider mixed states with definite parity, which will be choose as odd. A general pure state will be then a linear combination of single fermion states and three fermion states. Therefore, it can be written as

$$
|\psi\rangle=\sum_{i}^{4}\left(\alpha_{i} c_{i}^{\dagger}|0\rangle+\bar{\beta}_{i} c_{i}|\overline{0}\rangle\right),
$$

where $|\overline{0}\rangle=c_{1}^{\dagger} c_{2}^{\dagger} c_{3}^{\dagger} c_{4}^{\dagger}|0\rangle$ is the fully occupied state and $\boldsymbol{\alpha}, \boldsymbol{\beta}$ are four-dimensional complex vectors satisfying $|\boldsymbol{\alpha}|^{2}+|\boldsymbol{\beta}|^{2}=1$. It can be shown 31] that the eigenvalues of the generalized one-body density matrix $\rho^{\mathrm{qsp}}$ of such state are four-fold degenerate and given by

$$
f_{ \pm}=\frac{1 \pm \sqrt{1-C^{2}(|\psi\rangle)}}{2}
$$

where $C(|\psi\rangle)=2\left|\boldsymbol{\beta}^{\dagger} \boldsymbol{\alpha}\right|$ is the generalized Slater correlation measure, satisfying $0 \leq C \leq 1$. This result implies that there is always a quasiparticle basis in which the state (62) takes the normal form

$$
|\psi\rangle=\left(\sqrt{f_{+}} a_{1}^{\dagger}+\sqrt{1-f_{+}} a_{2}^{\dagger} a_{3}^{\dagger} a_{4}^{\dagger}\right)|0\rangle,
$$


with $|0\rangle$ denoting now the vacuum of the operators $a_{i}$. In terms of $C$ the entanglement entropy (14) becomes then

$$
S^{\mathrm{qsp}}(|\psi\rangle)=4 h\left(\frac{1+\sqrt{1-C^{2}(|\psi\rangle)}}{2}\right),
$$

so $C(|\psi\rangle)$ plays the role of a fermionic concurrence. As in the two-qubit case, for a mixed state $\rho$ the convex roof extension of $S^{\text {qsp }}$ can be similarly evaluated as

$$
S^{\mathrm{qsp}}(\rho)=4 h\left(\frac{1+\sqrt{1-C^{2}(\rho)}}{2}\right),
$$

where $C(\rho)$ is the convex roof extension of the pure state concurrence defined above 31]. This quantity vanishes iff $\rho$ can be written as a convex combination of particle or quasiparticle Slater determinants.

Let us now consider the state (55) with a maximally entangled state $|\psi\rangle=\frac{1}{\sqrt{2}}\left(a_{1}^{\dagger}+a_{2}^{\dagger} a_{3}^{\dagger} a_{4}^{\dagger}\right)|0\rangle$, which leads to $f_{ \pm}=1 / 2$. The fermionic concurrence is then $C(\rho)=$ $\operatorname{Max}[(7 w-3) / 4,0]$ [46] and therefore, Eq. (66) leads to

$$
S^{\mathrm{qsp}}(\rho)=\left\{\begin{array}{ll}
0 & w \leq 3 / 7 \\
4 h\left(\frac{4+\sqrt{7[1+w(6-7 w)]}}{8}\right) & w>3 / 7
\end{array} .\right.
$$

The information loss $I_{f}^{\mathrm{qsp}}(\rho)$ can be easily evaluated from Eq. (58), since all single particle levels have probability 1/2 of being occupied:

$$
I_{f}^{\mathrm{qsp}}(\rho)=4\left[2 f\left(\frac{3 w+1}{8}\right)-f\left(\frac{7 w+1}{8}\right)-f\left(\frac{1-w}{8}\right)\right] .
$$

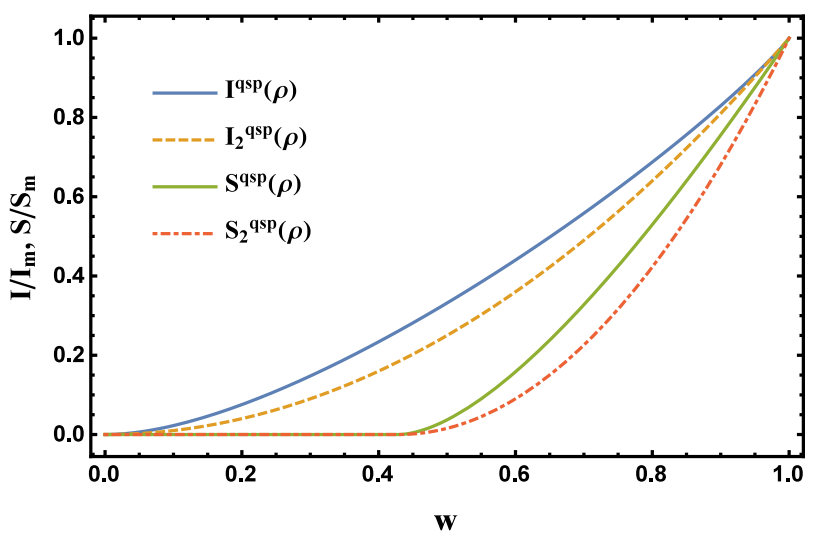

FIG. 1. Quadratic (dashed line) and von Neumann (solid line) information loss $I^{\mathrm{qsp}}(\rho)$ and entanglement of formation $S^{\mathrm{qsp}}(\rho)$ (normalized to their maximum values), as a function of $w$ for the mixture (55) with $n=4$ and definite odd parity, for a maximally entangled state $|\psi\rangle$.

Fig. 1 depicts this information loss in the von Neumann case $\left(f(p)=-p \log _{2} p\right)$ and in the quadratic case $(f(p)=2 p(1-p))$, together with the corresponding entanglement of formations $S^{\mathrm{qsp}}(\rho)$ and $S_{2}^{\mathrm{qsp}}(\rho)=4 C^{2}(\rho)$, as a function of $w$. While there is a threshold value, $w=3 / 7$, below which the state remains separable,
$I_{f}^{\mathrm{qsp}}(\rho)>0$ as soon as $\rho$ departs from the maximally mixed state, as given by Eq. (61), implying that the information loss detects "non classical" correlations beyond entanglement. In addition, it is worth noting that while $I_{2}^{\mathrm{qsp}}(\rho)$ is an upper bound to $S_{2}^{\mathrm{qsp}}(\rho)$ for the present states $\forall w \in(0,1)$, this is not strictly the case for the von Neumann based quantities, as $I^{\mathrm{qsp}}(\rho)<S^{\mathrm{qsp}}(\rho)$ for $w$ below but very close to 1 .

\section{Two fermion states}

We now assume $|\psi\rangle$ in (55) is a two-fermion state. These states are of the form

$$
|\psi\rangle=\frac{1}{2} \sum_{i, j} M_{i j} c_{i}^{\dagger} c_{j}^{\dagger}|0\rangle
$$

where $M$ is a $n \times n$ complex antisymmetric matrix satisfying $\frac{1}{2} \operatorname{Tr} M M^{\dagger}=1$. As shown by Zumino in [50], for any such matrix there is a unitary matrix $U$ such that $U^{\dagger} M \bar{U}=D$, where $D$ is a block diagonal matrix with $2 \times 2$ blocks of the form

$$
D_{k}=\sqrt{\lambda_{k}}\left(\begin{array}{cc}
0 & 1 \\
-1 & 0
\end{array}\right)
$$

with $\lambda_{k}$ a real number. With the corresponding unitary transformation $\boldsymbol{c}=U \boldsymbol{a}$ of the fermion operators, we can then split the sp space as $\mathcal{S}=\mathcal{S}_{\mathcal{A}} \oplus \mathcal{S}_{\mathcal{B}}$ and rewrite the state (69) in the normal form

$$
|\psi\rangle=\sum_{k} \sqrt{\lambda_{k}} a_{k(A)}^{\dagger} a_{k(B)}^{\dagger}|0\rangle .
$$

Eq. (71) is the Slater decomposition [23] of $|\psi\rangle$, a fermionic analogue (for two fermion states) of the Schmidt decomposition for distinguishable bipartite systems. The associated one-body density matrix is

$$
\rho^{\mathrm{sp}}=M M^{\dagger}=U D D^{T} U^{\dagger}
$$

which entails that the numbers $\lambda_{k}$ are its eigenvalues (twofold degenerate), as is also evident from Eq. (71). The Slater basis then diagonalizes $\rho^{\mathrm{sp}}$ (and hence $\rho^{\mathrm{qsp}}$, as there are here no pairing contractions). We then obtain $S^{\mathrm{qsp}}(|\psi\rangle)=S^{\mathrm{sp}}(|\psi\rangle)=2 \sum_{k} h\left(\lambda_{k}\right)$, which is the sum of the entanglement entropies of all sp modes of the Slater basis. We also have $S_{f}^{\mathrm{sp}}(|\psi\rangle)=2 \sum_{k} f\left(\lambda_{k}\right)+f\left(1-\lambda_{k}\right)$.

Let us now consider the the one-body information loss $I_{f}^{\mathrm{sp}}(\rho)$ for the ensuing mixture (55). Since the operators $a_{k(A)}, a_{k(B)}$ are the fermion operators where the onebody density matrix is diagonal, the measurement minimizing $I_{f}^{\mathrm{sp}}(\rho)$ (which coincides here with $I^{\mathrm{qsp}}(\rho)$ ) is that on the Slater basis of $|\psi\rangle$ and is then a function of the eigenvalues $\lambda_{k}$ :

$$
\begin{aligned}
I_{f}^{\mathrm{sp}}(\rho)= & 2 \sum_{k}\left[f\left(w \lambda_{k}+\frac{1-w}{d}\right)+f\left(w\left(1-\lambda_{k}\right)+\frac{1-w}{d}\right)\right. \\
& \left.-f\left(w+\frac{1-w}{d}\right)-f\left(\frac{1-w}{d}\right)\right]
\end{aligned}
$$


with $d=2^{n}$ in a grand canonical ensemble, $d=2^{n-1}$ in an even parity ensemble and $d=\frac{n(n-1)}{2}$ in a canonical ensemble. Present results are then formally similar to those obtained for similar mixtures in bipartite distinguishable systems [14], with the Slater basis replacing the local Schmidt basis.

\section{CONCLUSIONS}

We have discussed the problem of quantifying onebody discord-like correlations in general pure and mixed states of fermion systems, which may not have a fixed particle number (but which commute with the number parity). First, the correlation between a singleparticle mode and its orthogonal complement in the single-particle state space $\mathcal{S}$ is considered. The measurement of the occupancy of a single-particle mode is properly defined and an entropic measure of the loss of information due to this projective measurement is introduced. The sum over all modes in a given basis of $\mathcal{S}$ of this quantity, minimized over all such bases, is defined as the one-body information loss, a measure of the minimum loss of information due to an unread measurement. It is a non-negative quantity, invariant under arbitrary unitary transformations in $\mathcal{S}$, which vanishes if and only if the state of the system can be written as a convex combination of Slater determinants in a common basis of $\mathcal{S}$, i.e., if it remains invariant after the unread measurement of the occupancy of any sp level of this basis. For pure states it reduces to the fermionic entanglement entropy defined in 31]. These properties still hold if quasiparticle level occupancy measurements are allowed, in which case minimization is to be extended to all bases related through a Bogoliubov transformation. The defined quantities are then extended to more general entropic forms, including trace form entropies and quantum Renyi entropies. The general stationary condition to be satisfied by the minimizing measurement was also derived.

As application, we considered the mixture of a general pure state with the maximally mixed state for arbitrary space dimension. The minimum information loss was shown to be always reached for a measurement on the basis diagonalizing the generalized one-body density matrix, and to exhibit a universal quadratic initial increase with the mixing parameter, proportional to the quadratic entanglement entropy of the pure state. This fact implies that it can measure non classical correlations beyond entanglement, as explicitly verified for $\operatorname{dim}(\mathcal{S})=4$ (first non-trivial dimension for definite parity).

The approach can be applied in both canonical and grand-canonical ensembles, and for general states such as mixtures of independent qusiparticle states or vacua, being then suitable for studying correlations in strongly interacting fermion systems and requiring just a sp basis optimization. Its extension to more general partitions of the sp space may yield further insight into the structure of the state and is currently under investigation.

\section{Appendix: Entanglement of partitions of the fermionic single particle space}

We discuss here the entanglement associated to a general partition of a single particle (sp) space $\mathcal{S}$ of a fermionic system. Let us assume $\mathcal{S}$ is of dimension $n$, and consider a partition of $\mathcal{S}$ in two orthogonal subspaces $\mathcal{S}_{A}$ and $\mathcal{S}_{B}$, generated respectively by $m$ and $n-m$ orthogonal sp states of a given basis of $\mathcal{S}$, such that $\mathcal{S}=\mathcal{S}_{A} \oplus \mathcal{S}_{B}$. Any Slater determinant in this basis can then be written, except for a global phase, as

$$
\left|\psi_{s d}\right\rangle=\prod_{i \in \mathcal{S}_{A}}\left(c_{i}^{\dagger}\right)^{n_{i}} \prod_{j \in \mathcal{S}_{B}}\left(c_{j}^{\dagger}\right)^{n_{j}}|0\rangle \equiv|\mu \nu\rangle,
$$

where $n_{i(j)}=0,1$ is the occupation number of level $i(j)$ and $\mu=\left\{n_{i}, i \in \mathcal{S}_{A}\right\}, \nu=\left\{n_{j}, j \in \mathcal{S}_{B}\right\}$ indicate the collection of these numbers. The full set of states $|\mu \nu\rangle$, with $\mu=1, \ldots, 2^{m}, \nu=1 \ldots, 2^{n-m}$, form an orthonormal basis of the full (grand canonical) space of many fermion states in $\mathcal{S}$, of dimension $d=2^{n}$. Any pure fermion state $|\psi\rangle$ with definite number parity can then be written as

$$
|\psi\rangle=\sum_{\mu, \nu} C_{\mu \nu}|\mu \nu\rangle,
$$

where the sum is restricted to states $|\mu \nu\rangle$ of the same number parity as $|\psi\rangle$ (i.e., $|++\rangle$ or $|--\rangle$ states for $|\psi\rangle$ of even parity). This implies that the matrix $C$ of elements $C_{\mu \nu}$ is blocked in two submatrices $\left(C^{+}\right.$and $\left.C^{-}\right)$. After a singular value decomposition $C=U D V^{\dagger}$, with $D$ a "diagonal" $2^{m} \times 2^{n-m}$ matrix of non-negative elements $D_{k l}=\sigma_{k} \delta_{k l}$, and $U, V$ unitary matrices, we may rewrite this state as

$$
|\psi\rangle=\sum_{k} \sigma_{k}\left|k_{A} k_{B}\right\rangle
$$

with $\left|k_{A} k_{B}\right\rangle=\sum_{\mu, \nu} U_{\mu k} V_{\nu k}^{*}|\mu \nu\rangle$ orthonormal states. This is the Schmidt decomposition associated with this partition. Of course, $U$ and $V$ are also blocked, so that all states $\left|k_{A} k_{B}\right\rangle$ have the same number parity as $|\psi\rangle$.

The entanglement entropy associated with the previous partition is then

$$
E_{A B}=S\left(\rho_{A}\right)=S\left(\rho_{B}\right)=-\sum_{k} p_{k} \log _{2} p_{k},
$$

with $p_{k}=\sigma_{k}^{2}$ the eigenvalues of $C C^{\dagger}$ (or $C^{\dagger} C$ ) and

$$
\begin{aligned}
& \rho_{A}=\sum_{\mu, \mu^{\prime}}\left(C C^{\dagger}\right)_{\mu \mu^{\prime}}|\mu\rangle\left\langle\mu^{\prime}\left|=\sum_{k} p_{k}\right| k_{A}\right\rangle\left\langle k_{B}\right|, \\
& \rho_{B}=\sum_{\nu, \nu^{\prime}}\left(C^{\dagger} C\right)_{\nu \nu^{\prime}}|\nu\rangle\left\langle\nu^{\prime}\left|=\sum_{k} p_{k}\right| k_{B}\right\rangle\left\langle k_{B}\right|,
\end{aligned}
$$

the reduced states associated with subspaces $\mathcal{S}_{A}$ and $\mathcal{S}_{B}$, such that any observable $O_{A(B)}$ containing operators $c_{i}^{\dagger}$, $c_{i}$ with $i \in \mathcal{S}_{A(B)}$ can be obtained as

$$
\left\langle O_{A(B)}\right\rangle=\operatorname{Tr} \rho_{A(B)} O_{A(B)} .
$$


A Slater determinant in a given sp basis is then completely separable, in the sense that $E_{A B}=0$ for any bipartition $(m, n-m)$ of $\mathcal{S}$ in this basis. On the other hand, if a state is not a Slater determinant there is no sp basis in which it is completely separable. Note that $\rho_{A(B)}$ commutes with number parity but will contain in general eigenstates of both parities.

In particular, if $\mathcal{S}_{A}$ contains just a single state (say $k$ ), Eq. (A.1) becomes

$$
\begin{aligned}
|\psi\rangle & =\sum_{\nu} C_{0 \nu}|0 \nu\rangle+\sum_{\nu^{\prime}} C_{1 \nu^{\prime}}\left|1 \nu^{\prime}\right\rangle \\
& =\sqrt{p_{0}}\left|0 \psi_{0}\right\rangle+\sqrt{p_{1}}\left|1 \psi_{1}\right\rangle
\end{aligned}
$$

where $p_{i}=\sum_{\nu}\left|C_{i \nu}\right|^{2}$ and $\left|i \psi_{i}\right\rangle=\sum_{\nu} C_{i \nu}|i \nu\rangle / \sqrt{p_{i}}$ for $i=0,1$. Eq. (A.6), equivalent to (4), is the Schmidt decomposition of $|\psi\rangle$ since the states $\left|\psi_{0}\right\rangle$ and $\left|\psi_{1}\right\rangle$ have opposite parity and are therefore orthogonal. The ensuing entanglement (A.3) is then given by Eq. (8).

Let us now consider the entanglement of mixed states. If a partition of $\mathcal{S}_{B}$ in two subspaces $\mathcal{S}_{B_{1}}$ and $\mathcal{S}_{B_{2}}$ is made, we may define the associated entanglement of formation of $\rho_{B}, E_{B_{1} B_{2}}\left(\rho_{B}\right)$, as the minimum of the average entanglement entropies

$$
\sum_{\alpha} p_{\alpha} E_{B_{1} B_{2}}(|\alpha\rangle\langle\alpha|)=\sum_{\alpha} p_{\alpha} S\left(\rho_{B_{1}}^{\alpha}\right)
$$

over all decompositions $\rho_{B}=\sum_{\alpha} p_{\alpha}|\alpha\rangle\langle\alpha|$, with $p_{\alpha} \geq 0$, $\sum_{\alpha} p_{\alpha}=1, \rho_{B_{1}}^{\alpha}$ the reduced state of $B_{1}$ in the state $|\alpha\rangle$ and $|\alpha\rangle$ normalized many fermion states in $\mathcal{S}_{B}$, with the restriction (since $\left[\rho_{B}, P_{B}\right]=0$ ) that all states $|\alpha\rangle$ have definite number parity. This implies that $E_{B_{1} B_{2}}$ will be the average of the entanglement of formations for each parity, i.e., $E_{B_{1} B_{2}}\left(\rho_{B}\right)=p_{+} E_{B_{1} B_{2}}\left(\rho_{B}^{+}\right)+p_{-} E_{B_{1} B_{2}}\left(\rho_{B}^{-}\right)$ if $\rho_{B}=p_{+} \rho_{B}^{+}+p_{-} \rho_{B}^{-}$.

In particular, if $\mathcal{S}_{A}$ is a single state, Eq. (A.6) leads to

$$
\rho_{B}=p_{0}\left|\psi_{0}\right\rangle\left\langle\psi_{0}\left|+p_{1}\right| \psi_{1}\right\rangle\left\langle\psi_{1}\right|
$$

with $\left|\psi_{0}\right\rangle$ and $\left|\psi_{1}\right\rangle$ of opposite parity. Therefore, in this case the decomposition is unique and the entanglement of formation reads

$$
E_{B_{1} B_{2}}=p_{0} S\left(\rho_{B_{1}}^{0}\right)+p_{1} S\left(\rho_{B_{2}}^{1}\right)=S\left(B_{1} \mid A\right)
$$

where $S\left(B_{1} \mid A\right)$, equivalent to Eq. (22), denotes the conditional entropy of subsystem $B_{1}$ after a measurement of the single level of $A$ in the mixed state $\rho_{A B_{1}}$, in agreement with the general result of [39].

It is worth remarking that a general fermionic mixed state $\rho_{A}$ defined over a given sp space $\mathcal{S}_{A}$ (and commuting with $P$ ) can be purified in many ways, but in particular (and efficiently) by the addition of a complementary fermionic sp space $\mathcal{S}_{B}$, as is evident from the previous discussion. In a grand canonical context, if $\rho_{A}$ is, say, of rank $2^{n}$, with $2^{n} / 2$ eigenstates of each parity, it is sufficient to add $n$ orthogonal sp states generating an orthogonal sp space $\mathcal{S}_{B}$, and then form a pure state like (A.2) or (A.1), which should have a definite number parity if physically realizable. If all eigenstates of $\rho_{A}$ are of the same parity then $n+1$ sp states should be added and just many fermion states of definite parity should be considered.

The authors acknowledge support from CONICET (NG) and CIC (RR) of Argentina. Work supported by CIC and CONICET PIP 112201501-00732.
[1] C.H. Bennett et al, Phys. Rev. Lett. 70, 1895 (1993).

[2] M. A. Nielsen and I. L. Chuang, Quantum Computation and Quantum Information (Cambridge Univ. Press, Cambridge, UK, 2000).

[3] R. Josza and N. Linden, Proc. R. Soc. London, Sect. A 459, 2011 (2003);

[4] G. Vidal, Phys. Rev. Lett. 91, 147902 (2003).

[5] E. Knill, R. Laflamme, Phys. Rev. Lett. 815672 (1998).

[6] A. Datta, S.T. Flammia, C.M. Caves, Phys. Rev. A 72, 042316 (2005).

[7] K. Modi et al, Rev. Mod. Phys. 84, 1655 (2012).

[8] H. Ollivier, W.H. Zurek, Phys. Rev. Lett. 88017901 (2001)

[9] L.Henderson,V.Vedral J. Phys. A 346899 (2001); V.Vedral Phys. Rev. Lett. 90050401 (2003).

[10] W. H. Zurek, Phys. Rev. A 67, 012320 (2003).

[11] A. Datta, A. Shaji, C.M. Caves, Phys. Rev. Lett. 100, 050502 (2008).

[12] J. Oppenheim, M. Horodecki, P. Horodecki, R. Horodecki, Phys. Rev. Lett. 89180402 (2002).

[13] A. Streltsov, H. Kampermann, D. Bruß, Phys. Rev. Lett. 106, 160401 (2011).

[14] R. Rossignoli, N. Canosa, and L. Ciliberti, Phys. Rev. A
82, 052342 (2010); Phys. Rev. A 84, 052329 (2011).

[15] B. Dakić, V. Vedral, С̆. Brukner, Phys. Rev. Lett. 105 190502 (2010).

[16] M. Piani et al, Phys. Rev. Lett. 106, 220403 (2011).

[17] D. Girolami, T. Tufarelli, G. Adesso, Phys. Rev. Lett. 110, 240402 (2013).

[18] S. Luo, S. Fu, C.H. Oh, Phys. Rev. A 85, 032117 (2012).

[19] F.M. Paula, T.R. de Oliveira, M.S. Sarandy, Phys. Rev. A 87, 064101 (2013).

[20] T. Nakano, M. Piani, G. Adesso, Phys. Rev. A 88, 012117 (2013).

[21] F. Ciccarello, T. Tuffarelli, V. Giovannetti, New J. Phys. 16013038 (2014).

[22] F. Iemini, T. Debarba, R.O. Vianna, Phys. Rev. A 89, 032324 (2014).

[23] J. Schliemann, J.I. Cirac, M. Kus, M. Lewenstein, D. Loss, Phys. Rev. A 64, 022303 (2001).

[24] J. Schliemann, D. Loss, and A.H. MacDonald, Phys. Rev. B 63, 085311 (2001).

[25] K. Eckert, J. Schliemann, D. Bruß and M. Lewenstein, Ann. Phys. 299, 88 (2002).

[26] H.M. Wiseman and J.A. Vaccaro, Phys. Rev. Lett. 91, 097902 (2003). 
[27] C.V. Kraus, M.M. Wolf, J.I. Cirac, G. Giedke, Phys. Rev. A 79, 012306 (2009).

[28] F. Iemini, R.O. Vianna, Phys. Rev. A 87, 022327 (2013).

[29] M. Oszmaniec, J. Gutt, M. Kuś, Phys. Rev. A 90, 020302(R) (2014); M. Oszmaniec, M. Kuś, Phys. Rev. A 90, 010302(R) (2014); ibid 88, 0523328 (2013).

[30] G. Sárosi, P. Lévay, J. Phys. A 47, 115304 (2014); Phys. Rev. A 90, 052303 (2014); ibid 89, 042310 (2014).

[31] N. Gigena, R. Rossignoli, Phys. Rev. A 92042326 (2015).

[32] P. Zanardi, Phys. Rev. A 65, 042101 (2002).

[33] Y. Shi, Phys. Rev. A 67, 024301 (2003).

[34] N. Friis, A.R. Lee, and D.E. Bruschi, Phys. Rev. A 87, 022338 (2013).

[35] F. Benatti, R. Floreanini, U. Marzolino, Phys. Rev. A 89, 032326 (2014); X.M. Puspus, K.H. Villegas, F.N.C. Paraan, Phys. Rev. B 90, 155123 (2014).

[36] P. Ring, P. Schuck, The Nuclear Many-Body Problem (Springer, Berlin, 1980).

[37] N. Friis, New J. Phys. 18, 033014 (2016).

[38] R. Bhatia, Matrix Analysis (Springer, NY, 1997); A. Marshall and I. Olkin, Inequalities: Theory of Majorization and its Applications (Academic Press, 1979).

[39] M. Koashi, A. Winter, Phys. Rev. A 69022309 (2004).
[40] R. Horodecki, M. Horodecki, Phys.Rev. A 541838 (1996).

[41] M.A. Nielsen M. Kempe, Phys. Rev. Lett. 865184 (2001).

[42] R. Rossignoli, N. Canosa, Phys. Rev. A 66042306 (2002); Phys. Rev. A 67042302 (2003).

[43] M. Plenio, S. Virmani, Quantum Inf. Comp. 7, 1 (2007).

[44] N. Canosa, R. Rossignoli, Phys. Rev. Lett. 88170401 (2002)

[45] R. Filip, Phys. Rev. A 65, 062320 (2002); H. Nakazato et al, Phys. Rev. A 85, 042316 (2012); T. Tanaka, G. Kimura, H. Nakazato, Phys. Rev. A 87, 012303 (2013).

[46] N. Gigena, R. Rossignoli, Phys. Rev. A 90042318 (2014).

[47] C. Tsallis J. Stat. Phys. 52479 (1988); Introduction to non extensive statistical mechanics (Springer, Berlin 2009).

[48] A. Renyi, Probability Theory (North Holland, Amsterdam (1970)); C. Beck, F. Schlögl, Thermodynamic of Chaotic Systems (Cambridge Univ. Press, UK, 1993).

[49] N. Canosa, L.Ciliberti, R. Rossignoli, Entropy 171634 (2015).

[50] B. Zumino, J. Math. Phys. 31055 (1962). 